\title{
Aortic dilation, genetic testing, and associated diagnoses
}

\author{
Yuri A. Zarate, MD1, Elizabeth Sellars, MD¹, Tiffany Lepard, MS¹, \\ Xinyu Tang, $\mathrm{PhD}^{2}$ and $\mathrm{R}$. Thomas Collins II, $\mathrm{MD}^{3,4}$
}

Purpose: The aims of this study were to determine the genetic diagnoses most frequently associated with aortic dilation in a large population and to describe the results of genetic testing in the same.

Methods: A retrospective review of records from patients with known aortic dilation identified through an echocardiogram database was performed. During the study period, different chromosomal microarray platforms and molecular diagnostic techniques were used.

Results: A total of 715 patients (mean age, 9.7 years; $67 \%$ male) met study inclusion criteria. The overall frequency of underlying presumptive or confirmed genetic diagnoses was 17\% (125/715). Molecular evaluation for possible underlying aortopathy-related disorders was performed in $9 \%$ of patients $(66 / 715)$. Next-generation sequencing panels were performed in 16 patients, and pathogenic abnormalities were detected in 4 (25\%). Microarrays were conducted in $10 \%$ of patients $(72 / 715)$, with a total of 23 pathogenic copy-number variants identified in 19 patients (26\%). Marfan syndrome was the most frequently recognized genetic disorder associated with aortic dilation, but other cytogenetic abnormalities and associated diagnoses also were identified.

Conclusion: The differential diagnosis in patients with aortic dilation is broad and includes many conditions outside the common connective tissue disorder spectrum. A genetics evaluation should be considered to assist in the diagnostic evaluation.

Genet Med advance online publication 2 July 2015

Key Words: aortic dilation; bicuspid aortic valve; connective tissue disorders; genetic testing; Marfan syndrome

\section{INTRODUCTION}

Aortic dilation (AoD) at the level of the aortic root can be caused by a variety of congenital or acquired conditions that lead to weakening of the aortic wall. ${ }^{1-3}$ Congenital aneurysmal dilation has been associated with not only a number of congenital heart defects (CHDs) but also several syndromic connective tissue disorders (CTDs) such as Marfan syndrome, Loeys-Dietz syndrome, and Ehlers Danlos syndrome type IV, among others. In addition to the genes responsible for these genetic disorders, mutations in many other genes related to the structure and function of the aortic wall have been found in nonsyndromic familial forms of thoracic aortic aneurysms, such as familial thoracic aortic aneurysm and aortic dissection syndrome. ${ }^{4,5}$

The differential diagnosis of patients with AoD includes other genetic conditions. Chromosomal syndromes such as Turner syndrome and 22q11.2 deletion syndrome have been independently identified to be risk factors for AoD, apart from the presence of bicuspid aortic valve (BAV) or other CHDs known to be associated with de novo or postsurgical AoD and aortic dissection (i.e., conotruncal defects or ventricular septal defects). ${ }^{6-8}$ Patients with Noonan syndrome seem to be at a higher risk for annular AoD and aortic root aneurysms, whereas cases of thoracic aortic dissection have been reported in association with autosomal dominant polycystic kidney disease. ${ }^{5,9}$ Sinus of Valsalva aneurysms have also been described in patients with Klippel-Feil syndrome, cutis laxa, and Treacher-Collins syndrome. ${ }^{10}$

While the cornerstones for establishing a diagnosis continue to be a complete medical history, a detailed three-generation family history, and a comprehensive physical examination, there is great phenotypic overlap among CTDs. ${ }^{11}$ Clinicians often proceed with ordering molecular or cytogenetic studies in an attempt to reach a definitive diagnosis in hopes it will allow for improved counseling and will guide clinical monitoring and intervention.

The literature is limited with regard to the differential diagnosis of AoD, especially in the setting of recent advances in molecular and cytogenetic techniques. The aims of this study were to delineate the genetic diagnoses most frequently associated with AoD in a large population and describe the results of genetic testing in that population.

\section{MATERIALS AND METHODS}

After obtaining approval from the Arkansas Children's Hospital Institutional Review Board, a retrospective review of all patients with AoD evaluated at our center from 1 January 2009 to 1 July

\footnotetext{
${ }^{1}$ Section of Genetics and Metabolism, Department of Pediatrics, University of Arkansas for Medical Sciences, Little Rock, Arkansas, USA; ${ }^{2}$ Biostatistics Program,

Department of Pediatrics, University of Arkansas for Medical Sciences, Little Rock, Arkansas, USA; ${ }^{3}$ Division of Cardiology, Department of Pediatrics, University of Arkansas for Medical Sciences, Little Rock, Arkansas, USA; ${ }^{4}$ Department of Internal Medicine, University of Arkansas for Medical Sciences, Little Rock, Arkansas, USA. Correspondence: Yuri A. Zarate (yazarate@uams.edu)

Submitted 6 February 2015; accepted 19 May 2015; advance online publication 2 July 2015. doi:10.1038/gim.2015.88
} 
2013 was performed. Study subjects were identified by querying the Syngo Dynamics (version 9.0; Siemens Healthcare, Vienna, Austria) database for all echocardiography performed during the study period. Subjects were included if there was an echocardiographic diagnosis of at least mild dilation at the level of the aortic sinus of Valsalva or the ascending aorta as well as prior evaluation by a cardiologist and/or geneticist at our institution. Patients who had a history of heart transplantation or who had multiple echocardiograms with a lack of persistence of the diagnosis of AoD in at least two separate studies were excluded.

Echocardiographic measurements were made from inner edge to inner edge during midsystole and at the maximum diameter. Using the body surface area determined using the method described by Haycock et al., ${ }^{12} z$ scores for the aortic sinus of Valsalva and ascending aorta measurements available for each subject then were calculated using the regression equations by Warren et al. ${ }^{13}$ For the purposes of this study, AoD was described as mild for $z$ scores $\geq 2$ but $<4$, moderate for $z$ scores $\geq 4$ but $<6$, and severe for $z$ scores $\geq 6$.

Data abstracted from the medical records included age at echocardiography, age at diagnosis of AoD, sex, anatomic location of the AoD, aortic dimensions, anthropometric measurements, genetic evaluation, family history, suspected or confirmed genetic diagnoses, associated clinical findings, and concurrent CHDs, if present. When available, results of genetic testing also were reviewed. Family history was determined to be positive if first-degree relatives were affected with one of the following: a CHD, Marfan syndrome, another aortopathy (AoD, dissection, rupture, or aneurysm), or other CTD (Ehlers Danlos syndrome, Loeys-Dietz syndrome, or unknown).

During the study period, different chromosomal microarray platforms were used to analyze genomic DNA extracted from peripheral blood. Earlier patients had oligonucleotide-based platforms (Agilent Human Genome $44 \mathrm{~K}$ and $180 \mathrm{~K}$ Oligo array, Agilent Technologies, Santa Clara, CA; and 720K Oligo array, Roche NimbleGen, Madison, WI), whereas more recent cases were evaluated using the CytoscanHD whole-genome SNP array (Affymetrix, Santa Clara, CA). Whenever possible, microarray findings were confirmed by fluorescence in situ hybridization analysis.

Single-gene sequencing or sequential individual-gene testing was performed by Sanger sequencing in some patients. When performed, all coding exons of the ACTA2, COL3A1, COL5A1, COL5A2, FBN1, FBN2, MYH11, SMAD3, TGFBR1, and/or TGFBR2 genes were amplified by polymerase chain reaction. The amplified products then were sequenced and analyzed for sequence variations. In several other patients, next-generation sequencing (NGS) techniques were applied for testing. Targeted capture of all coding exons and exonintron junctions was followed by massively parallel sequencing of a combination of 10 to 31 of the following genes, depending on the performing laboratory: ABCC6, ACTA2, ACVR1, ADAMTS2, ADAMTSL4, ATP6VOA2, CBS, CHST14, COL1A1, COL1A2, COL2A1, COL3A1, COL5A1, COL5A2, COL9A1,
Table 1 Demographic and clinical characteristics of the study population

\begin{tabular}{lc} 
Characteristics & $\begin{array}{c}\text { All patients } \\
(\boldsymbol{n}=\mathbf{7 1 5})^{\mathrm{a}}\end{array}$ \\
\hline Male sex & $482(67 \%)$ \\
\hline Age at diagnosis of AoD (years) & $9.7 \pm 8.5(n=572)$ \\
Weight $(\mathrm{kg})$ & $44 \pm 29$ \\
Height $(\mathrm{m})$ & $1.4 \pm 0.4$ \\
\hline Body mass index $\left(\mathrm{kg} / \mathrm{m}^{2}\right)$ & $20.2 \pm 5.9$ \\
\hline Evaluated by genetics & $112(16 \%)$ \\
Family history & \\
Positive & $93(13 \%)$ \\
Negative & $303(42 \%)$ \\
Unknown & $319(45 \%)$ \\
BAV & $355(50 \%)$
\end{tabular}

Aortic data

Sinuses of Valsalva mean $z$ score

$3.5 \pm 1.1$

Sinuses of Valsalva dilation severity

Mild $260(75 \%)$

Moderate $\quad 74(21 \%)$

Severe $\quad 12(3 \%)$

Total 346

Ascending aorta mean z score

Ascending aorta dilation severity

Mild

$4.4 \pm 1.5$

$\begin{array}{lc}\text { Moderate } & 189(37 \%) \\ \text { Severe } & 75(15 \%) \\ \text { Total } & 512\end{array}$

Associated diagnoses

CHD

$501(70.1 \%)$

BAV

310

Idiopathic

$81(11.3 \%)$

Genetic causes

Chromosomal $\quad 52(7.3 \%)$

Marfan syndrome $\quad 48(6.7 \%)$

Other genetic $\quad 15(2.1 \%)$

VACTERL $\quad 5$

Shimke immuno-osseous dysplasia 2

Muscular dystrophy 2

Prune belly syndrome 1

Septo-optic dysplasia 1

Rothmund Thompson syndrome 1

Russel Silver syndrome

Arthrogryposis 1

Goldberg Shprintzen syndrome 1

Loeys-Dietz syndrome $\quad 6(0.8 \%)$

Other CTD $4(0.6 \%)$

FTAAD 3

Hypermobile EDS 1

Other nongenetic $8(1.1 \%)$

$\mathrm{AoD}$, aortic dilation; $\mathrm{BAV}$, bicuspid aortic valve; $\mathrm{CHD}$, congenital heart disease; CTD, connective tissue disorder; EDS, Ehlers Danlos syndrome; FTAAD, familial thoracic aortic aneurysm and aortic dissection syndrome.

asummary statistics are expressed as mean (standard deviation) for continuous variables and as frequency (as number (percentage)) for categorical variables. 


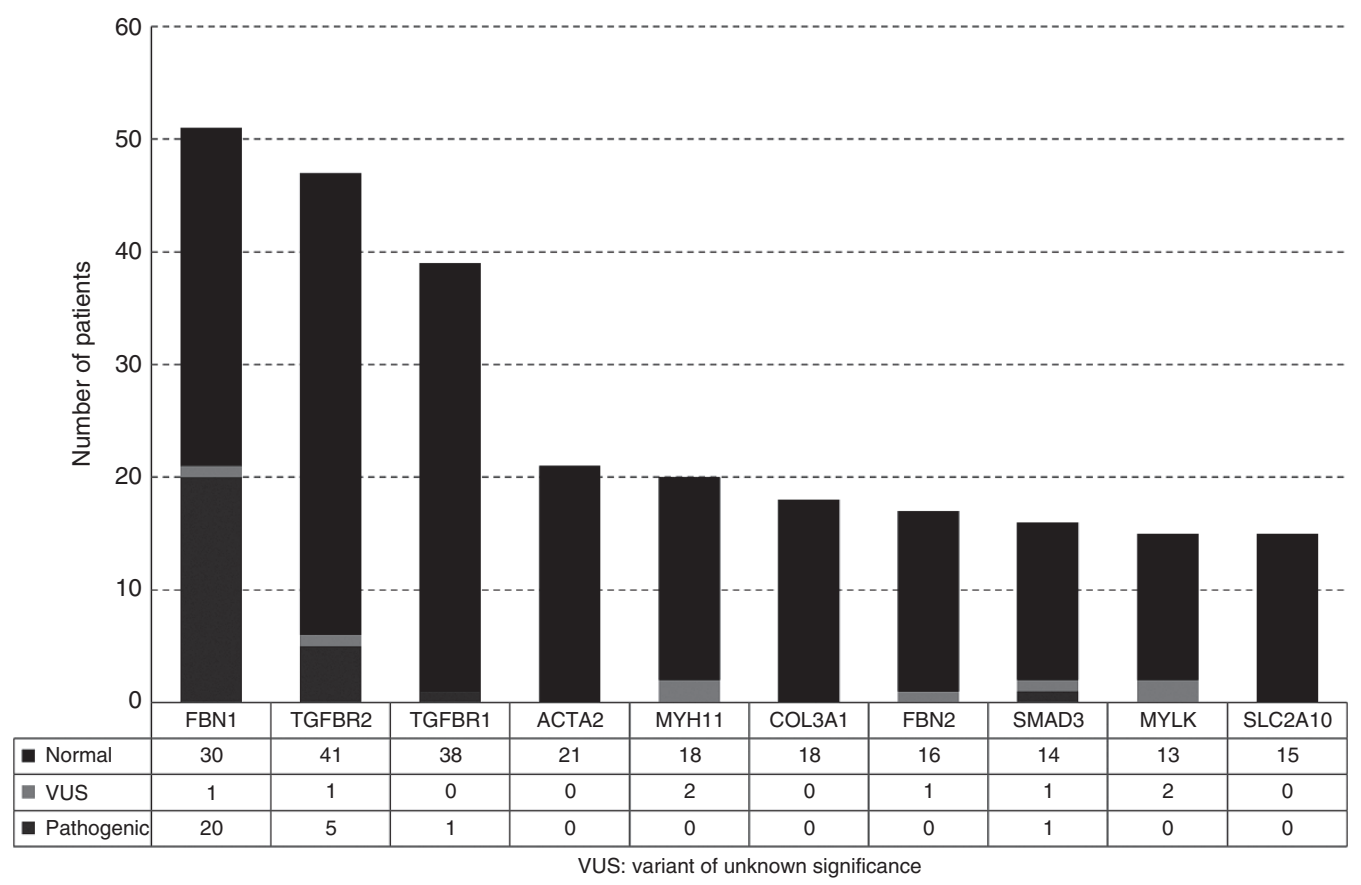

Figure 1 Sequencing results of the 10 most commonly tested genes in a population of 715 patients with aortic dilation.

COL11A1, ELN, FBLN5, FBN1, FBN2, FKBP14, LAMB1, MED12, MYH11, MYLK, NOTCH1, PRDM5, PKD2, PLOD1, SKI, SLC2A10, SLC39A13, SMAD3, SMAD4, TGFB2, TGFBR1, TGFBR2, TNXB, ZDHHC9, or ZNF469. Detected variants were confirmed by Sanger sequencing. In some cases deletion/duplication analysis was performed using a tiled, custom-designed comparative genomic hybridization array.

\section{RESULTS}

\section{Population description}

A total of 1,248 charts were reviewed, and data were collected on 715 patients who met study inclusion criteria (Table 1). The mean age at diagnosis of AoD for the entire cohort was 9.7 years (range, 1 day to 56 years). AoD was most often reported in the ascending aorta, whether alone $(369 / 715,52 \%)$ or in combination with the sinus of Valsalva $(143 / 715,20 \%)$. Isolated dilation of the sinus of Valsalva occurred in $203 / 715$ patients (28\%). The severity of AoD was mild in $59 \%$ of the total observations (508/858), regardless of the location.

\section{Molecular genetic testing for CTD}

In $70 \%$ of patients $(501 / 715)$ the $\mathrm{AoD}$ was presumed to be related to the presence of otherwise isolated BAV or other conotruncal heart defects, and therefore no further molecular genetic testing was ordered to evaluate the aortopathy. Of the remaining 214 cases, 50\% (107/214) underwent a genetic evaluation, and further molecular studies were conducted in $31 \%$ $(66 / 214)$, with single- or multigene testing for possible underlying aortopathy-related disorders (average five genes/patient). In the majority of patients $(53 / 66=80 \%)$ an underlying CTD was suspected by the clinician, whereas the remaining patient testing was performed because of a strong family history of aortopathy or because of the unexplained nature of the AoD. Overall, FBN1 was the single most commonly evaluated gene, followed by TGFBR2 and TGFBR1 (Figure 1).

The molecular evaluation was performed using NGS panels in 16 patients (average 14 genes/patient; range, 10-31 genes), 4 (25\%) of which had pathogenic mutations. There were eight variants of unknown significance (VUSs) found in six additional patients (38\%). Conversely, one or more genes were tested sequentially by Sanger sequencing in the remaining 50 patients (average two genes/patient; range, one to seven genes). Pathogenic mutations and VUSs were found in 23 patients (46\%) and 2 patients (4\%), respectively (Table 2 and Supplementary Table $S 1$ online).

\section{Molecular cytogenetic studies}

Microarrays were ordered in $10 \%$ of patients (72/715), the majority with oligonucleotide-based platforms (45/72, 63\%). Indications for the test to be ordered included developmental delay, cardiac or other congenital birth defects, dysmorphic features, growth retardation, or a combination of these. A total of 23 pathogenic copy-number variants were identified in 19 patients (26\%), whereas 16 VUSs were found in 15 patients (21\%) (Table 3; Supplementary Table S2 online).

\section{Other associated diagnoses}

The overall frequency of all the diagnoses encountered in this study is summarized in Table 1 . In this population, 48 patients $(6.7 \%)$ were diagnosed with Marfan syndrome, making it the most frequently recognized genetic disorder associated with AoD. For 19 patients, the diagnosis was confirmed with FBN1 mutations, whereas the remaining 29 patients were diagnosed on a clinical basis using the revised 
Table 2 Variants identified by Sanger sequencing and next-generation sequencing in patients with aortic dilation

\begin{tabular}{|c|c|c|c|c|c|c|}
\hline Methodology & Patient ID & Gene & Mutation & Classification & Exon & Phenotype \\
\hline \multirow[t]{17}{*}{ Sanger Sequencing } & 13 & TGFBR2 & c.1583G>T (p.Arg528Leu) & Pathogenic & 7 & $\begin{array}{l}\text { AoD, Skel, bifid } \\
\text { uvula, AT }\end{array}$ \\
\hline & 24 & FBN1 & c. $5788+5 G>A($ IVS $46+5 G>A$ & Pathogenic & $46-47$ & $A \circ D, E L$ \\
\hline & 50 & FBN1 & c. $6164-1 \mathrm{G}>\mathrm{C}(\mathrm{IVS} 49-1 \mathrm{G}>\mathrm{C})$ & Pathogenic & $49-50$ & $A \circ D, E L$ \\
\hline & 113 & TGFBR2 & c. $1258 G>A(p . G l y 420 A r g)$ & Pathogenic & 5 & $\begin{array}{l}\text { AoD, Skel, bifid } \\
\text { uvula, AT }\end{array}$ \\
\hline & 130 & FBN1 & c. $1571 \mathrm{delC}$ & Pathogenic & 12 & AoD, Syst \\
\hline & 229 & FBN1 & c.8056_8057delTG & Pathogenic & 64 & AoD, Syst \\
\hline & 343 & FBN1 & c.4716_4717delTC & Pathogenic & 37 & AoD, Syst, FH \\
\hline & 366 & TGFBR2 & c. $1031 \mathrm{G}>C$ (Trp344Ser) & Pathogenic & 4 & $\begin{array}{l}\text { AoD, Skel, bifid } \\
\text { uvula, AT }\end{array}$ \\
\hline & 428 & FBN1 & c.5513G>A (p.Gly1838Asp) & Pathogenic & 44 & AoD, Syst, FH \\
\hline & 434 & FBN1 & c.4286G>A (p.Cys1429Ser) & Pathogenic & 34 & $A \circ D, E L$ \\
\hline & 438 & FBN1 & c.203G>A (Cys68Tyr) & Pathogenic & 2 & AoD, Syst \\
\hline & 496 & FBN1 & c. $2638 \mathrm{G}>\mathrm{A}$ (p.Gly880Ser) & Pathogenic & 21 & AoD, Syst, FH \\
\hline & 523 & MYH11 & c.4604G>A (p.Arg1535GIn) & VUS & 33 & AoD \\
\hline & 530 & TGFBR1 & c.722C>T (p.Ser241Leu) & Pathogenic & 4 & AoD, Skel, Sz, ptosis \\
\hline & 536 & FBN1 & c.6635delA & Pathogenic & 54 & AoD, Syst, FH \\
\hline & 537 & TGFBR2 & c. $1657 \mathrm{~T}>\mathrm{A}$ (p.Ser553Thr) & VUS & 7 & $A \circ D, F H$ \\
\hline & 565 & FBN1 & c.4765T>C (p.Cys1589Arg) & Pathogenic & 38 & $A \circ D, E L$ \\
\hline & 182 & FBN1 & c.2638G>A (p.Gly880Ser) & Pathogenic & 21 & $A o D, E L$ \\
\hline & 245 & COL5A2 & c. $567-17 \mathrm{G}>\mathrm{T}$ & VUS & & AoD, Skel \\
\hline & 245 & $T N X B$ & c.11155C>T (p.Arg3719Trp) & VUS & 31 & AoD, Skel \\
\hline & 284 & FBN1 & c.266G>T (p.Cys89Phe) & Pathogenic & 3 & AoD, EL, FH \\
\hline & 401 & MYH11 & c.4235C>T (p.Ala1412Val) & VUS & 31 & AoD \\
\hline & 401 & MYLK & c.4165T>G (p.Phe1389Val) & VUS & 23 & AoD \\
\hline & 431 & FBN2 & c.460A>G (p.Met154Val) & VUS & 4 & $A \circ D, F H$ \\
\hline & 538 & FBN1 & c. 3586G>A (p.Val1 196Ile) & VUS & 29 & AoD \\
\hline & 609 & SMAD3 & c.803G>A (p.Arg268His) & VUS & 6 & AoD, Syst \\
\hline & 686 & SMAD3 & c.369dupT (p.Pro124fs) & Pathogenic & 1 & AoD, bifid uvula \\
\hline & 687 & $M Y L K$ & c. 2113 C>T (p.Arg705Cys) & VUS & 14 & AoD, Syst \\
\hline
\end{tabular}

AoD, aortic dilation; AT, arterial tortuosity; CP, cleft palate; cranio, craniosynostosis; EL, ectopia lentis; FH, positive family history; Skel, skeletal abnormalities; Syst, systemic features of Marfan syndrome; SZ, seizures; VUS, variant of unknown significance.

Ghent criteria $^{14}: 15$ patients with AoD and a systemic score >7; 6 patients with AoD and ectopia lentis; 5 patients with AoD, a systemic score $>7$, and a positive family history; and 3 more with AoD, ectopia lentis, and a positive family history. Meanwhile, only 6 patients $(0.8 \%)$ with Loeys-Dietz syndrome were identified (all with pathogenic mutations in either TGFBR1 or TGFBR2), and 4 more (0.6\%) were diagnosed with other CTDs. 
Table 3 Abnormal microarray results interpreted as pathogenic

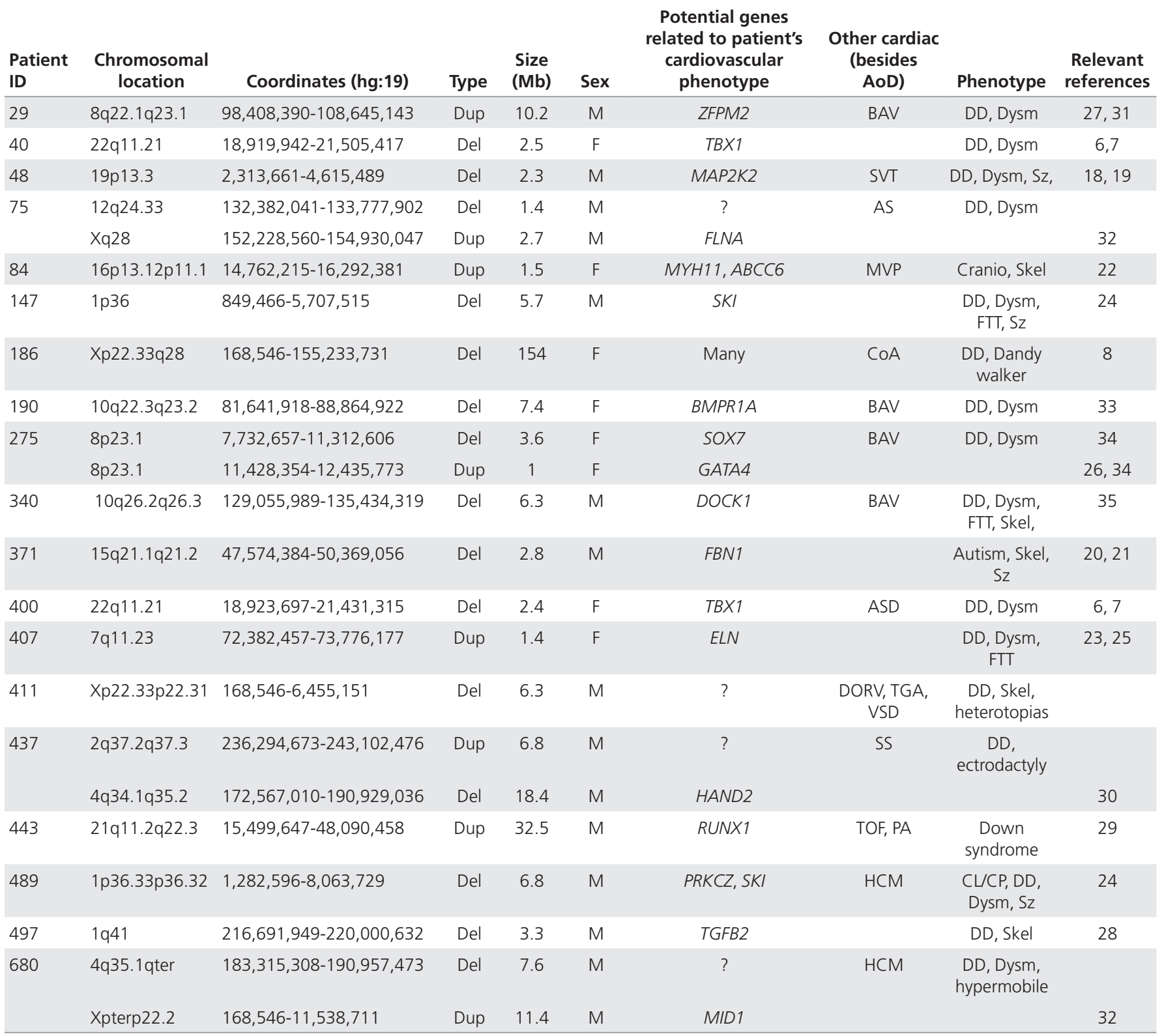

AoD, aortic dilation; AS, aortic stenosis; ASD, atrial septal defect; BAV, bicuspid aortic valve; CL/CP, cleft lip and palate; CoA, coarctation of the aorta; Cranio, craniosynostosis; DD, developmental delay; Del, deletion; DORV, double-outlet right ventricle; Dup, duplication; Dysm, dysmorphic features; F, female; FTT, failure to thrive; HCM, hypertrophic cardiomyopathy; M, male; MVP, mitral valve prolapse; PA, pulmonary atresia; Skel, skeletal abnormalities; SS, subaortic stenosis; SVT, supraventricular tachycardia; Sz, seizures; TGA, transposition of the great arteries; VSD, ventricular septal defect.

In addition to the 19 patients who had pathogenic microarray studies, 33 patients with AoD were identified as having chromosomal abnormalities through the use of conventional cytogenetic studies and fluorescence in situ hybridization techniques $(52 / 715,7 \%)$. In total, Turner syndrome was the most frequent (14 cases), followed by Down syndrome (9 cases), 22q11.2 deletion (7 cases), trisomies 13 and 18 ( 2 cases each), Williams syndrome ( 2 cases), and Jacobsen syndrome ( 1 case). Of interest, all patients with Turner syndrome had a CHD (11/14 with BAV (79\%)), but $13 / 38(34 \%)$ of the remaining patients with chromosomal abnormalities had otherwise structurally normal hearts on an echocardiogram (including two patients with 22q11.2 deletion).

Aortic dilation also was present in 15 patients (2\%) with a variety of other genetic conditions. Of the five patients with VACTERL association, only two had BAV. Structurally normal hearts were found in the two patients with Shimke immunoosseous dysplasia (both with pathogenic SMARCL1 mutations) and the two patients with unspecified muscular dystrophies.

An underlying etiology for AoD could not be identified in the remaining $11 \%$ of cases (81/715) despite additional comprehensive genetic testing such as microarrays (18 patients), 


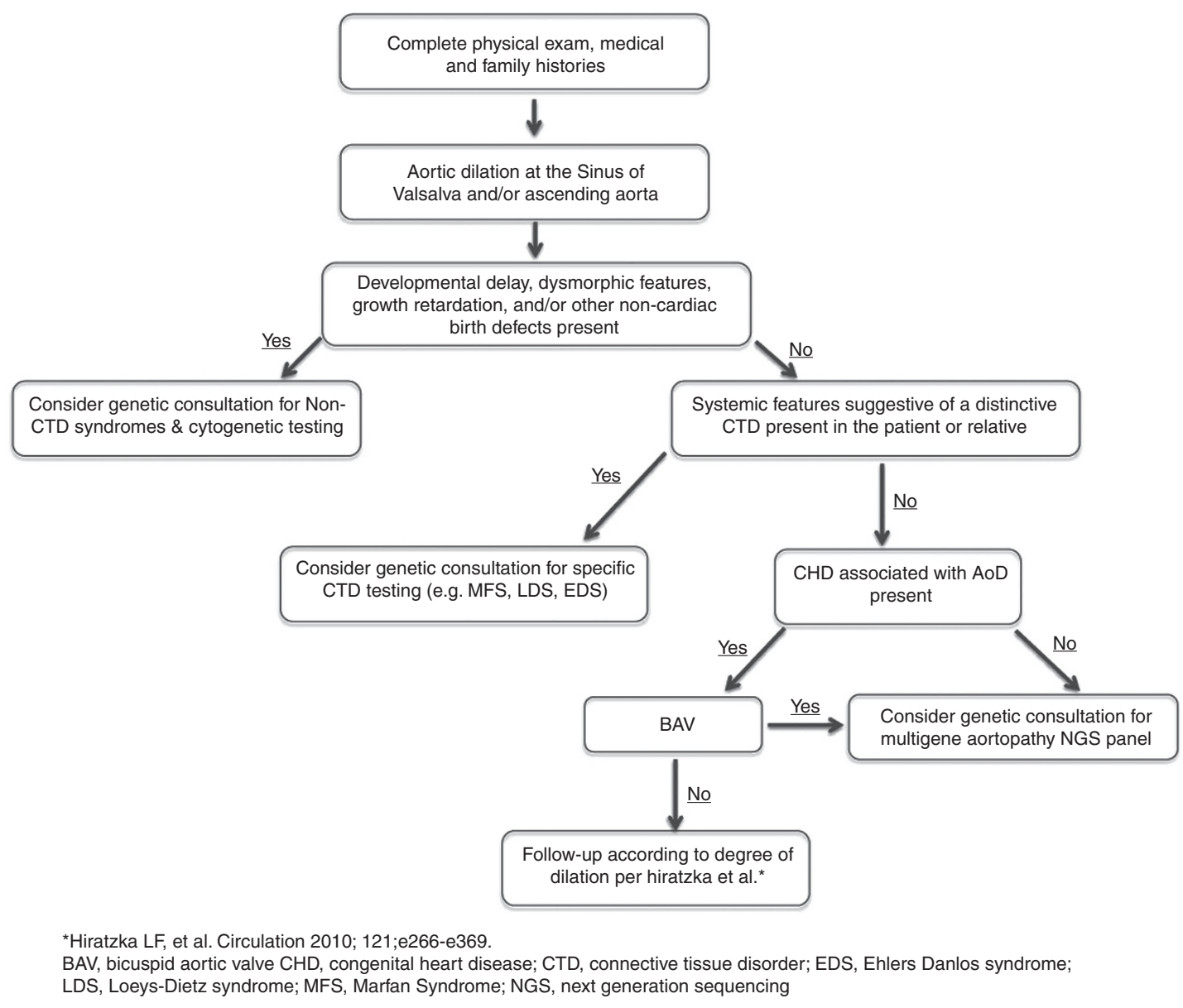

Figure 2 Proposed diagnostic evaluation of patients with aortic dilation.

connective-tissue gene panels (10 cases), or individual sequencing of one or more genes (25 patients). In this group of "idiopathic" AoD, only $22(27 \%)$ did not have other associated anomalies. The remaining 59 (73\%) with idiopathic AoD had other birth defects, developmental delay, skeletal anomalies, and/or dysmorphic features.

\section{DISCUSSION}

In this large, retrospective study of patients with AoD, the overall frequency of underlying presumptive or confirmed genetic diagnoses was $17 \%$. This small percentage, however, is likely an underestimate of the overall genetic component. The genetic nature of BAV, the large proportion of patients who remained undiagnosed despite additional genetic testing, and the limited genetic input provided for the majority of patients also need to be considered.

Over the past few years, NGS has emerged as a cost-effective alternative to sequential sequencing with traditional Sanger methods to detect mutations in genes associated with multigenic disorders. ${ }^{15}$ With the known phenotypic overlap among CTDs and the sometimes imperative need to provide better counseling and guide clinical monitoring and intervention, NGS panels have been designed to explore simultaneously several genes known to cause different CTDs. ${ }^{16,17}$ Including patients who had both NGS panels and conventional Sanger sequencing studies, molecular genetic studies for possible CTDs were performed in only $9 \%$ of patients. When those patients thought to have AoD caused by BAV or other conotruncal heart defects were excluded, the proportion of patients who underwent such studies was significantly higher. Conventional Sanger sequencing techniques for patients suspected clinically to have syndromic CTDs were helpful in reaching a diagnosis in close to half of those patients. Meanwhile, in this study only 16 patients underwent NGS panels, and pathogenic abnormalities were detected in only $25 \%$ of them. In retrospect, targeted mutation analysis rather than a panel could have been sufficient for three of these patients (two with FBN1 and one with TGFBR2 mutations) given their major findings (both patients with FBN1 also had ectopia lentis, and the patient with TGFBR2 had a bifid uvula and craniosynostosis). Our limited experience with these CTD-oriented panels also revealed a high frequency of reported VUSs (38\%) that should be interpreted carefully in the clinical context: Of the 11 reported VUSs, 6 were predicted to be damaging by at least 3 different in silico prediction tools.

In addition to the better-known syndromic CTDs, we were able to determine that, in the setting of AoD, chromosomal causes are identified just as frequently. In this population some of the diagnoses included major aneuploidies that should be easily 
recognizable, such as Down syndrome, Turner syndrome, and trisomies 13 and 18. There were many other smaller deletions and duplications identified that could not have been diagnosed without performing further molecular cytogenetic studies. Regardless of the presence of BAV or conotruncal defects, we were able to reaffirm previously reported relationships between AoD and several chromosomal abnormalities such as Turner syndrome, 22q11.2 deletion, 19p13.3 deletion, 16p13.1 duplication, $1 \mathrm{p} 36$ deletion, $15 \mathrm{q} 21.1$ deletion, and $7 \mathrm{q} 11.23$ duplication. $^{6-8,18-25}$ In many cases, in spite of lacking further supporting clinical evidence of a direct link between $\mathrm{AoD}$ and the genes involved in the remaining patients with cytogenetic abnormalities, other potential candidate genes with relationships to cardiac morphogenesis or pathology were identified (Table 3). ${ }^{26-35}$

We also were able to identify other less common genetic conditions in this population. VACTERL association, which was the most common, has been associated with a variety of heart defects, including BAV and conotruncal defects, but was not typically been associated with $\mathrm{AoD}$ in large series of patients. ${ }^{36}$ Likewise, Schimke immuno-osseous dysplasia, which was present in two patients in this cohort, has not been routinely associated with AoD, but rather with vascular disease secondary to impaired vascular elastogenesis. ${ }^{37}$ Given the single occurrence of the remaining conditions, a direct link is difficult to establish.

Based on the results presented here, we propose our current practice algorithm for patients with AoD (Figure 2). After a complete physical exam and careful review of the medical and family histories is completed, the evaluating physician can determine when a genetics consultation is recommended. In turn, the geneticist can help evaluate for potential CTDs or other syndromic conditions and determine the need for cytogenetic studies, targeted sequencing, or multigene panels at different stages of the process. The presence of concurrent cardiovascular defects (but no other systemic features) is weighted depending on the presence or absence of BAV, knowing the more recent evidence of its larger genetic component. ${ }^{38,39}$

This study is retrospective in nature and has several limitations. In some cases a single echocardiogram was available for analysis, and therefore it was not possible to confirm the persistence of AoD. Many patients with chromosomal abnormalities, as well those with other genetic diagnoses, also had BAV, a well-known risk factor for AoD. Therefore, in many cases it was not possible to determine the degree to which the aortopathy was related to BAV as opposed to the underlying, identified genetic abnormality. Finally, genetic services were underused in this cohort, explaining the limited availability of family history information in many cases. Despite these limitations, knowing that the literature is limited with regard to the differential diagnosis of AoD in the setting of recent advances in molecular genetic techniques, the results of this study are important and should help in the diagnostic evaluation of patients with AoD.

In summary, molecular cytogenetic studies, as well as conventional Sanger sequencing and NGS panels, often are performed in the evaluation of patients with AoD. Genetic diagnoses including chromosomal abnormalities, CTDs, and other syndromes, are found frequently in patients with AoD who do not have an isolated CHD. Our early experience with NGS panels for CTDs suggests that VUSs are seen frequently but require careful interpretation. Last, genetic services and evaluations are probably underused in the setting of AoD. Given the broad differential diagnosis for AoD that includes many conditions outside the common CTD spectrum, a referral for an evaluation by a geneticist is appropriate, especially in the context of other anomalies. Further, a geneticist could also assist in the diagnostic evaluation of the relatively high frequency of cases with an undetermined etiology.

\section{WEB RESOURCES}

SIFT, http://sift.bii.a-star.edu.sg/

PolyPhen-2, http://www.genetics.bwh.harvard.edu/pph2/

MutationTaster, http://www.mutationtaster.org/

Provean, http://provean.jcvi.org/index.php

MutPred, http://mutpred.mutdb.org/

\section{SUPPLEMENTARY MATERIAL}

Supplementary material is linked to the online version of the paper at http://www.nature.com/gim

\section{DISCLOSURE}

The authors declare no conflict of interest

\section{REFERENCES}

1. Boyer JK, Gutierrez F, Braverman AC. Approach to the dilated aortic root. Curr Opin Cardiol 2004; 19:563-569.

2. Jain D, Dietz HC, Oswald GL, Maleszewski JJ, Halushka MK. Causes and histopathology of ascending aortic disease in children and young adults. Cardiovasc Pathol 2011;20:15-25.

3. Hiratzka LF, Bakris GL, Beckman JA, et al.; American College of Cardiology Foundation/American Heart Association Task Force on Practice Guidelines; American Association for Thoracic Surgery; American College of Radiology; American Stroke Association; Society of Cardiovascular Anesthesiologists; Society for Cardiovascular Angiography and Interventions; Society of Interventional Radiology; Society of Thoracic Surgeons; Society for Vascular Medicine. 2010 ACCF/AHA/AATS/ACR/ASA/SCA/SCAI/SIR/STS/SVM guidelines for the diagnosis and management of patients with Thoracic Aortic Disease: a report of the American College of Cardiology Foundation/American Heart Association Task Force on Practice Guidelines, American Association for Thoracic Surgery, American College of Radiology, American Stroke Association, Society of Cardiovascular Anesthesiologists, Society for Cardiovascular Angiography and Interventions, Society of Interventional Radiology, Society of Thoracic Surgeons, and Society for Vascular Medicine. Circulation 2010;121:e266-e369.

4. Bisleri G, Bagozzi L, Muneretto C. Current evidence and insights about genetics in thoracic aorta disease. ScientificWorldJournal 2013;2013:962097.

5. Wu D, Shen YH, Russell L, Coselli JS, LeMaire SA. Molecular mechanisms of thoracic aortic dissection. J Surg Res 2013;184:907-924.

6. John AS, McDonald-McGinn DM, Zackai EH, Goldmuntz E. Aortic root dilation in patients with 22q11.2 deletion syndrome. Am J Med Genet A 2009;149A:939-942.

7. John AS, Rychik J, Khan M, Yang W, Goldmuntz E. 22q11.2 deletion syndrome as a risk factor for aortic root dilation in tetralogy of Fallot. Cardiol Young 2014;24:303-310.

8. Lopez L, Arheart KL, Colan SD, et al. Turner syndrome is an independent risk factor for aortic dilation in the young. Pediatrics 2008;121:e1622-e1627.

9. Cornwall JW, Green RS, Nielsen JC, Gelb BD. Frequency of aortic dilation in Noonan syndrome. Am J Cardio/ 2014;113:368-371.

10. Caruana M, Sheppard MN, Li W. Aneurysmal dilatation of the aortic sinuses of Valsalva - beyond Marfan syndrome: a single centre experience and review of the literature. Front Med 2014;8:419-426. 
11. Murphy-Ryan M, Psychogios A, Lindor NM. Hereditary disorders of connective tissue: a guide to the emerging differential diagnosis. Genet Med 2010;12: 344-354.

12. Haycock GB, Schwartz GJ, Wisotsky DH. Geometric method for measuring body surface area: a height-weight formula validated in infants, children, and adults. J Pediatr 1978;93:62-66.

13. Warren $A E$, Boyd ML, O'Connell C, Dodds L. Dilatation of the ascending aorta in paediatric patients with bicuspid aortic valve: frequency, rate of progression and risk factors. Heart 2006;92:1496-1500.

14. Loeys BL, Dietz HC, Braverman AC, et al. The revised Ghent nosology for the Marfan syndrome. J Med Genet 2010;47:476-485.

15. Metzker ML. Sequencing technologies - the next generation. Nat Rev Genet 2010;11:31-46.

16. Manase D, Manickaraj A, Al Turki S, Hurles ME, Mital S. High throughput exome coverage of clinically relevant cardiac genes. BMC Med Genomics 2014;7:67.

17. Wooderchak-Donahue WL, O'Fallon B, Furtado LV, et al. A direct comparison of next generation sequencing enrichment methods using an aortopathy gene panel- clinical diagnostics perspective. BMC Med Genomics 2012:5:50.

18. Nowaczyk MJ, Thompson BA, Zeesman S, et al. Deletion of MAP2K2/MEK2: a novel mechanism for a RASopathy? Clin Genet 2014;85:138-146.

19. Risheg $H$, Pasion $R$, Sacharow $S$, et al. Clinical comparison of overlapping deletions of 19p13.3. Am J Med Genet A 2013:161A:1110-1116.

20. Colovati ME, da Silva LR, Takeno SS, et al. Marfan syndrome with a complex chromosomal rearrangement including deletion of the FBN1 gene. Mol Cytogenet 2012;5:5.

21. Furtado LV, Wooderchak-Donahue W, Rope AF, et al. Characterization of large genomic deletions in the FBN1 gene using multiplex ligation-dependent probe amplification. BMC Med Genet 2011;12:119.

22. Kuang SQ, Guo DC, Prakash SK, et al.; GenTAC Investigators. Recurrent chromosome 16p13.1 duplications are a risk factor for aortic dissections. PLoS Genet 2011;7:e1002118.

23. Zarate YA, Lepard T, Sellars E, et al. Cardiovascular and genitourinary anomalies in patients with duplications within the Williams syndrome critical region: phenotypic expansion and review of the literature. Am J Med Genet $A$ 2014;164A:1998-2002.

24. Zaveri HP, Beck TF, Hernández-García A, et al. Identification of critical regions and candidate genes for cardiovascular malformations and cardiomyopathy associated with deletions of chromosome 1p36. PLoS One 2014;9:e85600

25. Parrott A, James J, Goldenberg P, et al. Aortopathy in the 7q11.23 microduplication syndrome. Am J Med Genet A 2015;167A:363-370.
26. Ballarati L, Cereda A, Caselli R, et al. Genotype-phenotype correlations in a new case of 8p23.1 deletion and review of the literature. Eur J Med Genet 2011;54:55-59.

27. De Luca A, Sarkozy A, Ferese $R$, et al. New mutations in ZFPM2/FOG2 gene in tetralogy of Fallot and double outlet right ventricle. Clin Genet 2011;80: 184-190.

28. Lindsay ME, Schepers D, Bolar NA, et al. Loss-of-function mutations in TGFB2 cause a syndromic presentation of thoracic aortic aneurysm. Nat Genet 2012;44:922-927.

29. Raveau M, Lignon JM, Nalesso V, et al. The App-Runx1 region is critical for birth defects and electrocardiographic dysfunctions observed in a Down syndrome mouse model. PLoS Genet 2012;8:e1002724.

30. Strehle EM, Yu L, Rosenfeld JA, et al. Genotype-phenotype analysis of $4 q$ deletion syndrome: proposal of a critical region. Am J Med Genet A 2012;158A:2139-2151.

31. Tan ZP, Huang C, Xu ZB, Yang JF, Yang YF. Novel ZFPM2/FOG2 variants in patients with double outlet right ventricle. Clin Genet 2012;82:466-471.

32. Tomita-Mitchell A, Mahnke DK, Struble CA, et al. Human gene copy number spectra analysis in congenital heart malformations. Physiol Genomics 2012:44:518-541.

33. van Bon BW, Balciuniene J, Fruhman G, et al. The phenotype of recurrent 10q22q23 deletions and duplications. Eur J Hum Genet 2011;19:400-408.

34. Wat MJ, Shchelochkov OA, Holder AM, et al. Chromosome 8p23.1 deletions as a cause of complex congenital heart defects and diaphragmatic hernia. Am J Med Genet A 2009;149A:1661-1677.

35. Yatsenko SA, Kruer MC, Bader PI, et al. Identification of critical regions for clinical features of distal 10q deletion syndrome. Clin Genet 2009;76: 54-62.

36. Cunningham BK, Hadley DW, Hannoush H, et al. Analysis of cardiac anomalies in VACTERL association. Birth Defects Res A Clin Mol Teratol 2013;97: 792-797.

37. Morimoto M, Yu Z, Stenzel P, et al. Reduced elastogenesis: a clue to the arteriosclerosis and emphysematous changes in Schimke immuno-osseous dysplasia? Orphanet J Rare Dis 2012;7:70.

38. Padang R, Bannon PG, Jeremy $R$, et al. The genetic and molecular basis of bicuspid aortic valve associated thoracic aortopathy: a link to phenotype heterogeneity. Ann Cardiothorac Surg 2013;2:83-91.

39. Pepe G, Nistri S, Giusti B, et al. Identification of fibrillin 1 gene mutations in patients with bicuspid aortic valve (BAV) without Marfan syndrome. BMC Med Genet 2014;15:23 